\title{
Thin Surface Layer of Plasma Treated Polyethylene
}

\author{
V. Kotál, ${ }^{1, a}$ P. Stopka, ${ }^{2}$ P. Sajdl, ${ }^{3}$ and V. Švorě́ík ${ }^{1}$ \\ ${ }^{1}$ Department of Solid State Engineering, Institute of Chemical Technology, Prague, Czech Republic \\ ${ }^{2}$ Institute of Inorganic Chemistry, Academy of Sciences of the Czech Republic, Rež, Czech \\ Republic \\ ${ }^{3}$ Department of Power Engineering, Institute of Chemical Technology, Prague, Czech Republic \\ a vladimir.kotal@vscht.cz
}

This paper reports on the effect of argon plasma on the high density polyethylene surface. The aim is to alter the surface in a manner and scale resulting in a stronger metal/polymer valence. The specimens are exposed to the direct current discharge, the irradiation time and power being variables. Electron paramagnetic resonance and X-ray photoelectron spectroscopy (EPR and XPS, respectively) are employed to determine the plasma effect. The surface wettability is studied by goniometry. The plasma treatment leads to radical generation and activation of such agents as oxygen, thus the surface wettability is significantly increased. The evolution of the treated surface in different media is studied. The influence of an increased oxygen concentration and the storage medium on the concentration gradient within the surface monolayers is proved. The EPR data show a gradual and very slow decrease in the number of radicals present on the treated surface after $2000 \mathrm{~h}$. Also evidence is given for partial dissolution of the treated surface in water.

Keywords: argon plasma, high density polyethylene, goniometry, X-ray photoelectron spectroscopy, electron paramagnetic resonance.

Introduction. Polymers have been applied successfully in many fields such as adhesion, biomaterials, protective coatings, friction and wear, composites, microelectronic devices, and thin-film technology. In general, special surface properties with regard to chemical composition, hydrophilicity, roughness, crystallinity, conductivity, lubricity, and cross-linking density are required for successful applications in various fields. However, the "raw-pristine" polymer surface is inert and the modification techniques need to be used [1].

Plasma treatment, which is known to modify chemical and physical states of the surface without altering the bulk properties, has become an important tool used in industry $[2,3]$. Plasma effect is versatile and strongly depends on the experimental conditions chosen. Take for example polyethylene, its plasma treatment leads to creation of new chemical groups, branching and crosslinking of macromolecules [2], and to formation of low molecular weight oxidized structures. Owing to ablation, the surface topography of the polymer is affected too. These alterations are also well known to result in the formation of reactive sites for the interaction with the metal atoms such as copper and aluminum. The metal polymer adhesion has been of highest interest recently and every attempt to elucidate their interaction is greatly appreciated.

The aim of this study is introduction of reactive sites to the high density polyethylene (HDPE) surface by argon plasma treatment. Further, the evolution of wettability, radical concentration, and chemical structure is thoroughly investigated. The surface wettability is studied by goniometry. X-ray photoelectron spectroscopy (XPS) is carried out to observe the surface chemical structure and electron paramagnetic resonance spectroscopy is employed for determination of the radical number. The experiment and the abovementioned methods yield a complex insight into the evolution of the HDPE plasma treated surface. 
Experimental. Polymer and Plasma Parameters Specification. Oriented HDPE in the form of $50 \mu \mathrm{m}$ thick foils was used in the present experiment. The foils were supplied by Granitol Ltd., Czech Republic. The samples were treated in a direct current discharge generated using Balzers SCD 050 device. The further discussed plasma effect was obtained under the following conditions (gas purity $99.997 \%$ and the flow rate $0.3 \mathrm{l} / \mathrm{s}$, pressure $10 \mathrm{~Pa}$, electrode distance $50 \mathrm{~mm}$ and its area $48 \mathrm{~cm}^{2}$, chamber volume approx. $1000 \mathrm{~cm}^{3}$, plasma volume $240 \mathrm{~cm}^{3}$, and power $8.3 \mathrm{~W}$ ). The treated polymer samples were stored under laboratory conditions, exposed to ambient atmosphere.

Diagnostic Methods. The contact angle, characterizing the surface wettability, was measured using distilled water at room temperature with a Kernco G-1 goniometer (Japan). The "static" contact angle dependence on the time after treatment was obtained [4].

An Omicron Nanotechnology ESCAProbeP spectrometer was used to observe the treated surface. The dimensions of the area analyzed were $2 \times 3 \mathrm{~mm}$. The X-ray source was monochromated at $1486.7 \mathrm{eV}$. The spectra were measured stepwise with a step in binding energy of $0.05 \mathrm{eV}$. In order to understand the cause forthe decrease in the oxygen content within several surface monolayers, the spectra were collected at six angles between the detector and the surface normal (ARXPS). The data were processed by the CasaXPS program.

The concentration of free radicals was determined using an electron paramagnetic resonance spectroscopy with an $\mathrm{x}$-band spectrometer of type Elexsys E-540, Bruker-Biospin with a relative error of $10 \%$. The samples were placed in a quartz tube and measured at room temperature. The experimental conditions were as follows: the magnetic field range $600 \mathrm{mT}$, sweep time $180 \mathrm{~s}$, magnetic modulation $0.4 \mathrm{mT}$, field modulation $100 \mathrm{kHz}$. The standards $\mathrm{Mn} / \mathrm{ZnS}$ and $\mathrm{Cr} / \mathrm{MgO}$ were used for the g-factor calibration and for quantitative evaluation of the spectra. Identification and determination of signals were performed by comparison with the standards.

Results and Discussion. Goniometry. The dependence of the water contact angle on the plasma treatment time is shown in Fig. 1. The time after the plasma treatment is a parameter of the curves. The higher the treatment time the lower the contact angle, namely: the angle decreases from $100^{\circ}$ (pristine HDPE) to $10^{\circ}$ (240 s treated HDPE). The increasing time after the plasma treatment leads to an increase in the contact angle. The increase is more distinct for longer plasma treatments. As has been reported in a recent study [5], the present measurements confirm the dependence of the contact angle (wettability) on the time after the Ar plasma treatment. The cause for this is the diffusion of the low-mass oxidized fragments and orientation of the polar groups towards the specimen bulk and this phenomenon is referred to as hydrophobic recovery [6, 7].

Electron Paramagnetic Resonance Spectroscopy (EPR). The number of radicals formed on the surface was monitored by the EPR. Figure 2 shows the number of radicals for samples stored in different "media." The "water" sample was stored in water for 12 hours, and then dried and examined. The "air" sample was kept in an ambient atmosphere. The lower number of radicals for a "water" sample results from the storage in water, which caused the removal of low-molecular-weight oxidized material from the treated surface [8]. This material contains a portion of the introduced radicals. Figure 2 also clearly shows a slow decrease in the number of radicals during storage. The free radical centers are "trapped" inside the crosslinked layer and are of low chemical reactivity, even if the surface is exposed to water [9].

X-ray Photoelectron Spectroscopy. The chemical structure of the plasma treated HDPE stored subsequently in air or water was examined using the XPS. It was reported that the surface of the Ar plasma treated HDPE contains groups of pristine $\mathrm{PE}\left(-\mathrm{CH}_{2}\right)$ and oxygen introduced during the treatment $(-\mathrm{C}=\mathrm{O},-\mathrm{COO}$, and $-\mathrm{COC}-)$ [5]. 


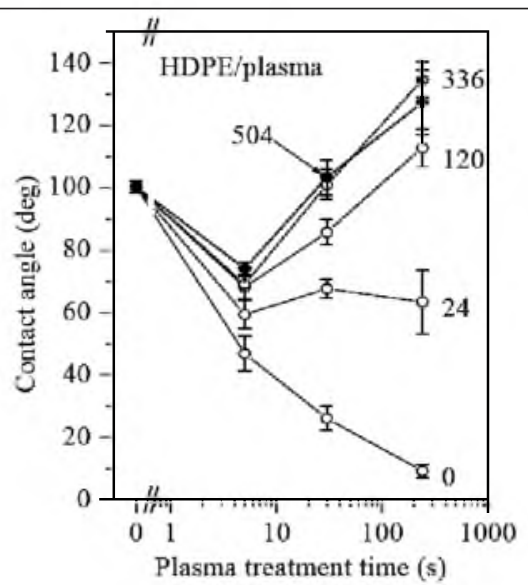

Fig. 1

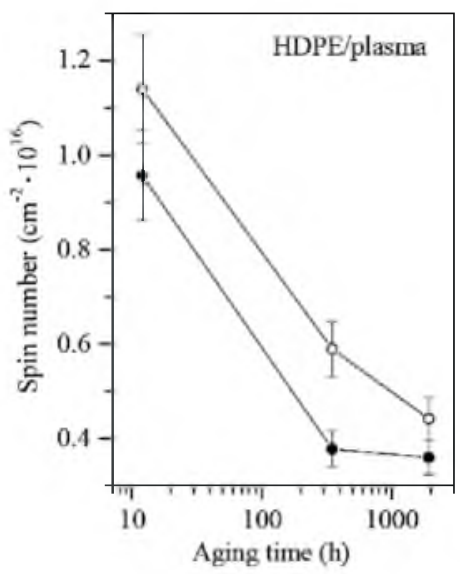

Fig. 2

Fig. 1. Evolution of the contact angle dependence on the plasma treatment time. The numbers represent hours elapsed after the treatment.

Fig. 2. Dependence of spin number of the plasma treated samples on time after the treatment. The samples were treated successively stored $12 \mathrm{~h}$ in water $(\bullet)$ resp. air $(\bigcirc)$ and measured.

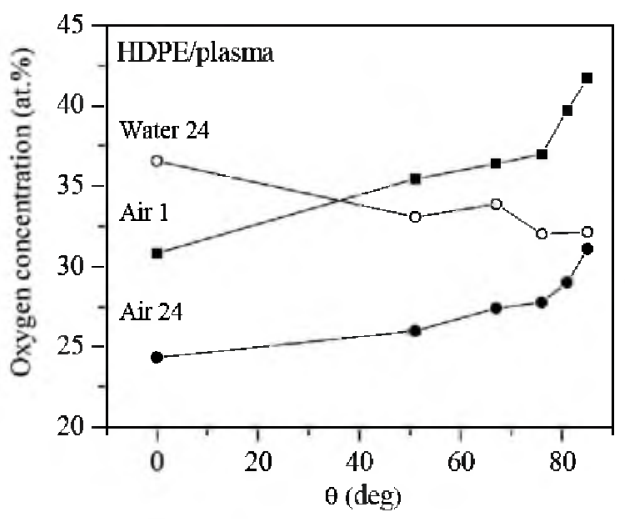

Fig. 3. The dependence of oxygen concentration on the detector to surface normal angle. The samples were plasma treated and preceding the measurement stored in air for $1 \mathrm{~h}$ (Air 1) and $24 \mathrm{~h}$ (Air 24). The sample (Water 24) was stored $24 \mathrm{~h}$ in water.

In the EPR study it we found that a portion of the treated surface is dissolved during storage in water. In order to confirm this result and also to learn more about the evolution of the first surface layers (within approx. $5 \mathrm{~nm}$ ) after the treatment, the angle-resolved XPS has been carried out $[10,11]$. Figure 3 shows the dependence of the oxygen concentration on the angle between the surface normal and the detector. The higher the angle, the thinner layer is studied, i.e., an angle of $80^{\circ}$ allows studying the structure of the surface monolayers. Figure 3 shows that the oxygen concentration in the samples treated and stored in air for $1 \mathrm{~h}$ and $24 \mathrm{~h}$ (Air 1 and Air 24, respectively) decreases towards the bulk of the sample. It has already been stated that the surface is oxygenated during the treatment. The post treatment oxygen incorporation is rather uncertain and some authors are in favor of it [12] while others are not [13]. What is worth noticing is that the oxygen concentration of the "Air 24" sample is lower than that of "Air 1." This has been shown by goniometry, the results of which proved increasing hydrophobic character after the treatment, i.e., during aging. Another important conclusion made from the ARXPS data is that the oxygen concentration in the water stored sample "Water 24" at high angles is 
lower than that in the "Air 1"sample. This suggests that a portion of the surface, especially of the oxidized material, is dissolved in water. This has been confirmed by the EPR in this work, as well as by the IR spectroscopy of the material dissolved in water [8]. Finally, the only explanation for the increase in the concentration of oxygen with a decrease in the angle for the "Water 24" sample is that the oxygen concentration increases into the depth of the material(within the $\mathrm{nm}$ scale). At a depths of the order of $10 \mathrm{~nm}$ we expect a sharp decrease in oxygen concentration. This is confirmed by Rutherford back scattering (RBS) analyses carried out on this sample [8].

Conclusions. The effect of the HDPE treatment in the Ar plasma discharge on its properties has been studied by different techniques. We have proved that the dischargeinduced surface alterations lead to an immediate increase in the surface wettability. Moreover, this effect is not permanent and the wettability decreases during the time after treatment. The EPR data show a gradual and very slow decrease in the number of radicals present on the treated surface; partial dissolving of the treated surface in water is also observed. The backbone of this report is the XPS observations, which revealed an increased oxygen concentration within the treated surface. Furthermore, it has been proved that the water storage causes an increase in the oxygen concentration gradient within the surface monolayers. On the contrary, when the sample is stored in air, the oxygen gradient decreases.

Acknowledgments. This work was supported by the GA ASCR under the project KAN400480701 and Ministry of Education of the CR under research program No. LC 06041.

1. C. M. Chan, T. M. Ko, and H. Hiraoka, Surf. Sci. Rep., 24, 3 (1996).

2. M. R. Wertheimer, A. C. Fozza, and A. Hollander, Nucl. Instrum. Meth. B, 151, 65 (1999).

3. P. K. Chu, J. Y. Chen, L. P. Wang, and N. Huang, Mater. Sci. Eng. R, 36, 143 (2002).

4. M. A. Grunlan, N. S. Lee, F. Mansfeld, et al., J. Polym. Sci., 44, 2551 (2006).

5. V. Švorčík, V. Kotál, P. Slepička, et al., Nucl. Instrum. Meth. B, 244, 365 (2006).

6. F. Truica-Marasescu, P. Jedrzejowski, and M. R. Wertheimer, Plasma Process. Polym., 1, 153 (2004).

7. S. Guimond and M. R. Wertheimer, J. Appl. Polym. Sci., 94, 1291 (2004)

8. V. Švorčík, V. Kotál, P. Slepička, et al., Polym. Deg. Stab. (submitted).

9. M. Kuzuya, T. Kawaguchi, M. Nakanishi, and T. Okuda, J. Chem. Soc. Faraday Trans., 82. 1441 (1986).

10. S. Oswald, R. Reiche, M. Zier, et al., Appl. Surf. Sci, 252, 3 (2005).

11. P. J. Cumpson, J. Elec. Spec. Rel. Phenomena, 73, 25 (1995).

12. M. Kuzuya, S. Kondo, M. Sugito, and T. Yamashiro, Macromolecules, 31, 3230 (1998).

13. O. Ochiello, Proc. of the 7th Int. Conf. on SIMS (1990), p. 789. 